\title{
Newcastle diseases virus strain V4UPM displayed oncolytic ability against experimental human malignant glioma
}

\begin{abstract}
Introduction: Newcastle disease virus (NDV) is a virus of paramyxovirus family and lately has been studied for the treatment of cancer in human. In this study, we successfully determined the oncolysis potential of NDV vaccine, V4UPM tested on the human glioblastoma multiform cell line (DBTRG.05MG) and human glioblastoma astrocytoma cell line (U-87MG) in vitro and in vivo. The V4UPM strain is a modified V4 strain developed as thermostable feed pellet vaccine for poultry. Objective: The objectives of this study were mainly to evaluate the cytolytic effect and subsequently determine the brain tumor regression potential induced by this strain in athymic mice model. Methodology and results: V4UPM, the avirulent strain of NDV, was propagate and screened for the cytolytic activity towards DBTRG.05MG and U-87MG using MTT assay. The inhibition concentration 50\% (IC50) values by monolayer method measured at hour 72 were 23 and $9 \mathrm{HAU} / \mathrm{ml}$, respectively. Further study was carried out to observe an apoptosis of the infected cells by AO/PI staining and revealed the apoptosis features of the treated cells. Subcutaneous human brain tumors grown on the nude mice were treated by V4UPM at IC80 and complete regression of U87MG-bearing tumor mice was observed. TUNEL assay analysis of treated tumor tissues from treated mice showed an occurrence of apoptosis. Conclusion: From this study, NDV strain V4UPM inhibits the proliferation of experimental human gliomas in tissue culture and IC80 at $520 \mathrm{HAU}$ V4UPM gives potent effect to induced tumor regression and apoptosis in malignant gliomas.
\end{abstract}

Keyword: Apoptosis; Glioblastoma; In vivo; NDV; V4UPM 